\title{
Streptomycin-Induced Steven-Johnson Syndrome in a HIV Sero-Positive Patient with Tuberculosis: A Case Report
}

\author{
Echendu Adinma, Nkiru N. Ezeama, Chukwuma D. Umeokonkwo
}

Department of Community Medicine, Nnamdi Azikiwe University Teaching Hospital, Nnewi, Nigeria.

Email: drechenduadinma@yahoo.com

Received June 24 $4^{\text {th }}$ 2011; revised July 25 ${ }^{\text {th }}, 2011$; accepted August 20 ${ }^{\text {th }}, 2011$.

\begin{abstract}
Severe cutaneous hypersensitivity reactions to anti-tuberculosis medication are rare and have been attributed mainly to thiacetazone. A case of streptomycin-induced Steven-Johnson Syndrome in a patient with TB-HIV co-infection which ended in death is reported.
\end{abstract}

Keywords: Streptomycin-Induced Steven-Johnson Syndrome, HIV Sero-Positive, Tuberculosis

\section{Introduction}

Steven-Johnson Syndrome (SJS) is a rare, acute and potentially fatal immune-complex hypersensitivity reaction that often presents as a life-threatening medical emergency. SJS and its more severe variant, Toxic Epidermal Necrolysis (TEN) are almost always caused by medications although viral infections and malignancies have been reported [1].

\section{Case Report}

An 18 year old female patient presented with main complaints of fever, chills, itchy generalised body rash, exfoliation of the lips and oral cavity, swelling and reddening of her eyes and sore throat, one week after commencing category two anti-tuberculosis regimen consisting of rifampicin, isoniazid, pyrazinamide and ethambutol (RHZE) in fixed-dose combinations and intramuscular injections of streptomycin. She was diagnosed as having an allergic reaction and placed on oral antihistamines and antibiotics. She represented to the clinic four days later with worsening of her symptoms and was attended to by a different doctor. On examination, an erythematous papular rash was observed on her face, trunk and extremities and there were blisters and ulcers on her lips and oral mucosa with drooling of saliva. The nasal and genital mucosae were also involved and the conjunctiva was inflamed with a thick purulent discharge (Figures 1 and 2).

The patient had been diagnosed seropositive to HIV two years prior to presentation with a baseline CD4 count of 320 cells $/ \mathrm{ml}$. She had completed eight months of category one regimen for sputum smear-positive pulmonary tuberculosis consisting of rifampicin, isoniazid, pyrazinamide and ethambutol in fixed-dose combinations and was declared cured following negative sputum smears at $5^{\text {th }}, 7^{\text {th }}$ and $8^{\text {th }}$ months of tuberculosis treatment. Two months later she had a relapse of smear-positive PTB for which she was then placed on the category two regimen of RHZE+ intramuscular streptomycin. The patient was not yet on antiretroviral therapy. Based on the clinical history and clinical examination, a diagnosis of StevenJohnson syndrome secondary to streptomycin was made and all anti-tuberculosis drugs were discontinued. She was admitted and started on triamcinolone $40 \mathrm{mg}$ daily, chlorpheniramine $4 \mathrm{mg}$ twice daily, ciprofloxacin 500 mg twice daily and intravenous infusions. On the third day of treatment the patient became dehydrated with a high- grade fever (temperature $39^{\circ} \mathrm{C}$ ). By the sixth day of treat- ment she developed frequent vomiting and diarrhoea with worsening odynophagia and dysphagia. She died on the ninth day.

\section{Discussion}

Steven-Johnson Syndrome (SJS) is a rare, acute immunecomplex hypersensitivity reaction characterised by sheetlike skin and mucosal loss. It is potentially fatal with a mortality rate of $5 \%-15 \%$ [2] and often presents as a life-threatening medical emergency. SJS and its more 


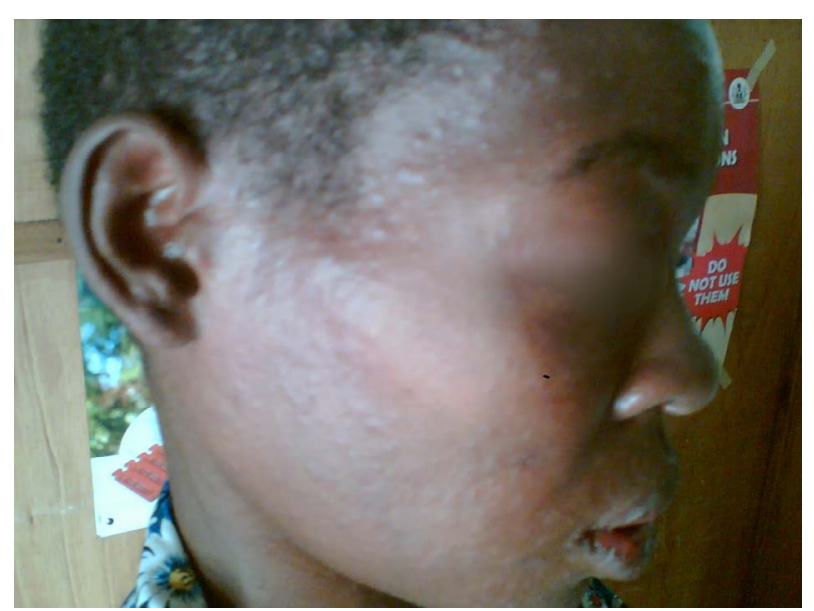

Figure 1. Erythematous papular rash on the face of the patient.

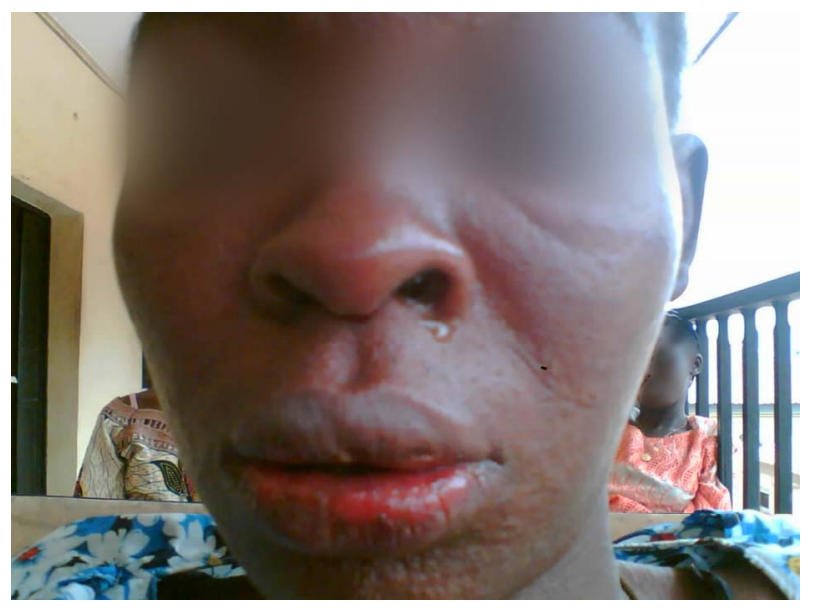

Figure 2. Conjunctival injection with purulent eye discharge, inflammation of nasal and oral mucosae, blistering and ulceration of lips.

severe variant, Toxic Epidermal Necrolysis (TEN) are almost always caused by medications although viral infections and malignancies have been reported; over 200 medications have been implicated in SJS with sulphonamide antibiotics being the most common inducing agents ${ }^{1}$, of which cotrimoxazole has been found to be the mostly commonly implicated in some studies [3,4]. SJS has become increasingly reported among patients co-infected with tuberculosis and the human immunodeficiency virus (HIV) who are on anti-tuberculosis chemotherapy [5]. The most commonly implicated drugs are thiacetazone, isoniazid and ethambutol [6,7]. Streptomycin is a rare cause of SJS; isolated cases of streptomycin-induced SJS have been reported $[8,9]$ and a rare case of streptomycin-induced TEN reported in 2004 [10]. The patient in this report had twice been treated for TB, the second time requiring addition of streptomycin according to the guide- lines for tuberculosis control in Nigeria. The lack of any reaction to the other anti-tuberculosis drugs in the 8-month long category I regimen strongly indicates a cause-effect relationship between streptomycin and SJS. Considering that HIV infection increases the risk for hypersensitivity reactions [11], it is unknown whether SJS in this case was caused solely by streptomycin or by streptomycin influenced by the concomitant HIV infection.

Management of SJS requires identifying and removing the offending drug. It may be difficult to identify the particular inducing agent and may need a step-wise exposure test. Treatment is essentially supportive and is similar to management of severe burns victims as skin damage may be quite extensive; close attention must be paid to maintaining hydration, nutritional support, pain management and preventing complications such as fluid and electrolyte imbalance, sepsis and synerchia formation in the eyes. Patients who die from SJS are often as a result of major complications such as septicemia, pneumonia, fluid and electrolyte imbalance leading to renal insufficiency [3]. Those who survive may end up with long term or even permanent complications such as blindness.

\section{Conclusions}

SJS requires a high index of suspicion by health workers and knowledge about the condition and mode of treatment. This patient may have survived if therapy was instituted earlier in the illness or if more aggressive measures of therapy and monitoring had been undertaken. Health workers who prescribe streptomycin for tuberculosis must be extra vigilant.

\section{REFERENCES}

[1] V. Ngan, A. Oakley and D. Dyall-Smith, "Stevens Johnson Syndrome and Toxic Epidermal Necrolysis," New Zealand Dermatological Society Incorporated, 2009. http://dermnetnz.org/nzds.html

[2] R. M. Patel and Y. S. Marfatia, "Clinical Study of Cutaneous Drug Eruptions in 200 Patients,” Indian Journal of Dermatology, Venereology and Leprology, Vol. 74, No. 4, 2008, p. 430. doi:10.4103/0378-6323.42883

[3] D. M. Thappa and R Kumari, "Steven-Johnson Syndrome,” Drug Alert, Vol. 2, No. 1, 2006, pp. 24-27.

[4] P. D. Ghislain and J. C. Roujeau. "Treatment of Severe Drug Reactions: Stevens-Johnson Syndrome, Toxic Epidermal Necrolysis and Hypersensitivity Syndrome," Dermatology Online Journal, Vol. 8, No. 1, 2002, p. 5.

[5] J. Wirrima and A. Harris, "Stevens-Johnson Syndrome during Anti-tuberculosis Chemotherapy in HIV-Seropositive Patients: Report on Six Cases,” East African Medical Journal, Vol. 68, No. 1, 1991, pp. 64-66.

[6] C. S. Dukes, J. Sugarman, J. P. Cegielski, G. J. Lallinger 
and D. H. Mwakyusa, "Severe Cutaneous Hypersensitivity Reactions during Treatment of Tuberculosis in Patients with HIV Infection in Tanzania," Tropical and Geographical Medicine, Vol. 44, No. 4, 1992, pp. 308311.

[7] C. Chintu, C. Luo, G. Bhat, M. Raviglione, H. DuPont and A. Zumla, "Cutaneous Hypersensitivity Reactions Due to Thiacetazone in the Treatment of Tuberculosis in Zambian Children Infected with HIV-I," Archives of Disease in Childhood, Vol. 68, No. 5, 1993, pp. 665-668. doi:10.1136/adc.68.5.665

[8] S. K. Sarkar, S. D. Purohit, T. N. Sharma, M. P. Chawla and D. N. Gupta, "Stevens-Johnson Syndrome Caused by Streptomycin,” Tubercle, Vol. 63, No. 2, 1982, pp. 137138. doi:10.1016/S0041-3879(82)80052-4
[9] P. R. Gupta, S. D. Purohit, S. K. Gupta, S. K. Sarkar and T. N. Sharma, "Stevens Johnson Syndrome Induced by Streptomycin: A Case Report,” Lung India, Vol. 2, No. 2, 1984, pp. 201-202.

[10] H. Hmouda, C. Laouani-Kechrid, M. Nejib Karoui, M. Denguezli, R. Nouira and G. Ghannouchi, "A Rare Case of Streptomycin-Induced Toxic Epidermal Necrolysis in a Patient with Tuberculosis: A Therapeutic Dilemma,” The Annals of Pharmacotherapy, Vol. 39, No. 1, 2005, pp. 165-168.

[11] S. A. Coopman, R. A. Johnson, R. Platt and R. S. Stern, "Cutaneous Disease and Drug Reactions in HIV Infection,” The New England Journal of Medicine, Vol. 328, 1993, pp. 1670-1674.

doi:10.1056/NEJM199306103282304 